\title{
Extremely low frequency magnetic field induces human neuronal differentiation through NMDA receptor activation
}

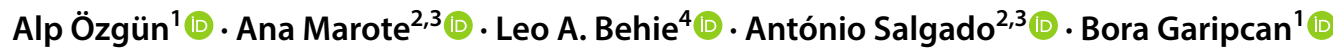

Received: 5 May 2019 / Accepted: 9 July 2019

(c) Springer-Verlag GmbH Austria, part of Springer Nature 2019

\begin{abstract}
Magnetic fields with different frequency and intensity parameters exhibit a wide range of effects on different biological models. Extremely low frequency magnetic field (ELF MF) exposure is known to augment or even initiate neuronal differentiation in several in vitro and in vivo models. This effect holds potential for clinical translation into treatment of neurodegenerative conditions such as autism, Parkinson's disease and dementia by promoting neurogenesis, non-invasively. However, the lack of information on underlying mechanisms hinders further investigation into this phenomenon. Here, we examine involvement of glutamatergic $\mathrm{Ca}^{2+}$ channel, N-methyl-D-aspartate (NMDA) receptors in the process of human neuronal differentiation under ELF MF exposure. We show that human neural progenitor cells (hNPCs) differentiate more efficiently under ELF MF exposure in vitro, as demonstrated by the abundance of neuronal markers. Furthermore, they exhibit higher intracellular $\mathrm{Ca}^{2+}$ levels as evidenced by c-fos expression and more elongated mature neurites. We were able to neutralize these effects by blocking NMDA receptors with memantine. As a result, we hypothesize that the effects of ELF MF exposure on neuronal differentiation originate from the effects on NMDA receptors, which sequentially triggers $\mathrm{Ca}^{2+}$-dependent cascades that lead to differentiation. Our findings identify NMDA receptors as a new key player in this field that will aid further research in the pursuit of effect mechanisms of ELF MFs.
\end{abstract}

Keywords Extremely low frequency magnetic field (ELF MF) $\cdot$ Neuronal differentiation $\cdot$ N-methyl-D-aspartate (NMDA) receptor $\cdot$ Human neural progenitor cells (hNPCs)

\section{Abbreviations}

ADAM10 A disintegrin and metalloproteinase AMPA Alpha-amino-3-hydroxy-5-methyl-4-isoxazole
propionic acid

António Salgado and Bora Garipcan share senior authorship.

António Salgado

asalgado@med.uminho.pt

$\triangle$ Bora Garipcan

bora.garipcan@boun.edu.tr

1 Institute of Biomedical Engineering, Bogazici University, Istanbul, Turkey

2 Life and Health Sciences Research Institute (ICVS), School of Medicine, University of Minho, Braga, Portugal

3 ICVS/3B's-PT Government Associate Laboratory, University of Minho, Braga/Guimarães, Portugal

4 Canada Research Chair in Biomedical Engineering (Emeritus), Schulich School of Engineering, University of Calgary, 2500 University Drive NW, Calgary, AB, Canada
CREB Cyclic AMP-responsive element-binding protein

DAPI 4-6-diamidino-2-phenylindole-dihydrochloride

EGFR Epidermal growth factor receptor

ELF MF Extremely low frequency magnetic field

hNPCs Human neural progenitor cells

mT MilliTesla

NMDA N-methyl-D-aspartate

\section{Introduction}

External stimuli that can trigger molecular events in biological media may prove valuable for both research applications and possible clinical tools. One prominent example is the development of optogenetic methods which enable optically triggering molecular cascades using light-sensitive proteins (Anikeeva 2016). However, these techniques usually require intracranial implantation of light delivery set ups as visible and near-infrared light is heavily absorbed by physiological 
media (Iseri and Kuzum 2017). Moreover, dependence on transgenically expressed light-sensitive proteins renders their clinical translation currently impossible (Chow and Boyden 2013; Gilbert et al. 2014). Consequently, vigorous efforts for finding new molecular manipulation strategies that can bypass the restrictions of optogenetics have been underway in recent years. The ability of magnetic fields to permeate tissues virtually undisturbed (Salvador et al. 2007; Hardwick et al. 2014; Klomjai et al. 2015) makes them the strongest candidate in this pursuit (Nimpf et al. 2017).

The field of magnetogenetics targets proteins that may be sensitive to external magnetic stimuli, such as IscA1 and ferritin, in an attempt to control ion channels (Qin et al. 2015; Wheeler et al. 2016; Stanley et al. 2014). Unfortunately, these promising results were proven to be impossible to occur via the suggested routes by very simple calculations (Anikeeva and Jasanoff 2016) as well as experimental results proving that the observed effects were not due to magnetic field exposure alone (Long and Zhang 2018). These findings necessitate a complete re-evaluation of this newly emerging field.

ELF MFs, on the other hand, produce influences on a wide array of native biological models without any transfected magneto-sensitive proteins. Furthermore, these effects appear under exposure to much weaker magnetic fields, on the level of several milliTeslas (mT) which present no cytotoxic or genotoxic effects (Boorman et al. 1997; Pettenati et al. 2018). There is already growing concern about the effects of exposure to ELF MFs as epidemiological (Davanipour et al. 2007; Zhou et al. 2012) and experimental (Consales et al. 2012, 2018) findings point to possible associations with neurodegenerative diseases. However, efforts are being made to identify useful effects under controlled exposure conditions. An increase in viability and collagen synthesis was observed in osteoblasts under the effects of $75-\mathrm{Hz}$ magnetic fields as well as an increase in angiogenesis factors (Saliev et al. 2014). Interestingly, $50 \mathrm{~Hz}$ was commonly accepted as the frequency for promoting neuronal differentiation and successfully used in studies on mesenchymal and neural stem cell lines (Cuccurazzu et al. 2010; Cho et al. 2012; Kim et al. 2013; Ma et al. 2014, 2016). Exposure to the same frequency also enhanced the efficiency of induced pluripotent stem cell reprogramming (Baek et al. 2014). It is important to realize here that responses to magnetic field exposure varies widely depending on parameters such as frequency, exposure duration (Ma et al. 2014; Yu et al. 2014) and cell type (Nikolova et al. 2005; Ma et al. 2014). These variables offer promising options in possible bio-modulatory applications (Maziarz et al. 2016).

NMDA receptors are glutamatergic ion channels that display high $\mathrm{Ca}^{2+}$ permeability (Chang and Kuo 2008). Unlike other glutamate-gated channels such as alpha-amino-3-hydroxy-5-methyl-4-isoxazole propionic acid (AMPA) and kainate receptors, NMDA receptor activation occurs slowly and activates $\mathrm{Ca}^{2+}$-dependent cascades to regulate neuronal processes such as synaptic plasticity (Blanke and VanDongen 2009; Iacobucci and Popescu 2017). Channel opening requires binding of glutamate and glycine to discrete binding sites. $\mathrm{A} \mathrm{Mg}^{2+}$ ion resides on a third binding site within the pore called a $\mathrm{Mg}^{2+}$ block which needs to be removed by the change in membrane potential during depolarization as a prerequisite for $\mathrm{Ca}^{2+}$ transition through the channel (Collingridge and Watkins 1994). NMDA receptor blockers such as memantine, ketamine, MK-801 and phencyclidine block the channel opening to prevent $\mathrm{Ca}^{2+}$ influx (Johnson and Kotermanski 2006; Parsons and Gilling 2007; Song et al. 2018). Memantine was utilized in this work as a lowaffinity NMDA receptor antagonist (Temme et al. 2018) to avoid possible toxicity effects that may arise during the long incubation period of the experiments.

One particularly important application here will be the effects of magnetic fields on neural cell differentiation which we hypothesize may prove to be a useful tool in treatment of neurodegenerative diseases especially since the effect is reproducible in in vivo models (Cuccurazzu et al. 2010). However, designing such a tool requires the knowledge of exact mechanisms of this effect to be able to tune field parameters for targeting specific molecular pathways. Unfortunately, discussions on these mechanisms in the literature are mostly postulations derived from the limited data we currently have which largely consist of changes in the expression levels of some proteins (Kim et al. 2013; Baek et al. 2014; Choi et al. 2014; Ma et al. 2016).

In the pursuit of effect mechanisms, the most commonly identified effect is the elevation of intracellular $\mathrm{Ca}^{2+}$ concentration in vivo and in vitro (Walleczek 1992; Karabakhtsian et al. 1994; Morgado-Valle et al. 1998; Fanelli et al. 1999; Zhou et al. 2002; Lisi et al. 2006; Manikonda et al. 2007; Sun et al. 2016). Concomitant effects of $\mathrm{Ca}^{2+}$ influx such as increased expression levels of c-fos and c-myc (Karabakhtsian et al. 1994) along with augmented protein kinase $\mathrm{A}$, protein kinase $\mathrm{C}$ and calcineurin activities (Manikonda et al. 2007) were also determined. Enhancement of neuronal differentiation under ELF MF exposure was previously linked to cyclic AMP-responsive element-binding protein (CREB) phosphorylation which could be traced back to epidermal growth factor receptor (EGFR) activation through Akt signaling in the upstream (Park et al. 2013). Still, no proven rationale exists about the starting point of the cascade that is triggered by the ELF MF exposure and results in neuronal differentiation. Considering the clues mentioned above, the NMDA receptor, a relatively unexplored candidate as a $\mathrm{Ca}^{2+}$ gateway in this field, may be the starting point in the cascade. NMDA receptor activation results in $\mathrm{Ca}^{2+}$ influx and activation of $\mathrm{Ca}^{2+}$-dependent pathways (Papadia and Hardingham 2007) which agrees with the elevation of 
intracellular $\mathrm{Ca}^{2+}$ under ELF MF exposure. It is also known that NMDA receptor activation heavily promotes neuronal differentiation (Yoneyama et al. 2008), as NMDA receptor signaling is a major regulator of proliferation and differentiation of neural progenitor cells (Joo et al. 2007; Jansson and Akkerman 2014). The hypothesis is further reinforced by the fact that the aforementioned EGFR-Akt-CREB cascade is stimulated by NMDA receptor activation at multiple points, namely; Akt phosphorylation is known to be triggered by NMDA treatment in vitro and EGFR phosphorylation can be enhanced by NMDA receptor activation through upregulation of a disintegrin and metalloproteinase 10 (ADAM10) (Tang et al. 2012).

Previous studies on effects of ELF EMF on NMDA receptors report altered ligand binding (Manikonda et al. 2007) and transient elevations in subunit expression ( $\mathrm{Li}$ et al. 2014; Kazemi et al. 2018) but do not address possible effects on activation. Considering the above information, it is tempting to hypothesize NMDA receptors to be the starting point of ELF MF effects on neural progenitors.

In this study, we explore possible involvement of NMDA receptors in the effect of ELF MFs on neuronal differentiation. We exposed differentiating hNPCs to $50 \mathrm{~Hz}, 1 \mathrm{mT}$ ELF $\mathrm{MF}$, as these are the most commonly utilized parameters for neuronal differentiation in the literature (Cui et al. 2017). We demonstrate that $50 \mathrm{~Hz}$ ELF MF exposure during neuronal differentiation enhances the levels of neuronal markers, c-fos and neurite outgrowth. These effects are completely reversed by treatment with an NMDA receptor antagonist, revealing a heavy NMDA receptor dependence. Implications and possible processes that lead to these findings are discussed. Our current results provide a robust affirmation to our hypothesis as all previously reported effects of ELF MF exposure are eliminated by memantine treatment. Furthermore, to the best of our knowledge, human neural progenitor cells (hNPCs) have never been used as an in vitro model of neuronal differentiation for ELF MF studies before, which makes the findings more relevant to ELF MF effects on human neural tissue.

\section{Materials and Methods}

\section{Cell culture and ELF MF exposure}

hNPCs were a kind gift from Prof. Leo A. Behie (University of Calgary, Calgary, Canada). Cells were isolated from the telencephalon region of a 10 week post-conception fetus according to the protocols and strict ethical guidelines previously established and approved by the Conjoint Health Research Ethics Board (CHREB, University of Calgary, Canada; ID: E-18786) guidelines (Mendez et al. 2002). Frozen neurospheres of hNPCs were thawed at $37^{\circ} \mathrm{C}$ and transferred into a T25 cell culture flask (Nunc) in PPRF-h2 medium (Baghbaderani et al. 2010). Two days later, neurospheres were dissociated by trituration and re-seeded in fresh PPRF-h2 medium to allow reformation and growth of spheres. During 14 days of growth, $40 \%$ of medium was refreshed every other day. For differentiation, neurospheres were enzymatically dissociated $(0.25 \%$ Trypsin-EDTA, GIBCO) and transferred to differentiation medium consisting of Neurobasal A (Thermo Fisher) supplemented with $10 \mathrm{ng} / \mathrm{mL}$ bFGF (Sigma Aldrich), 2\% B-27 supplement (Thermo Fisher) and 0.5\% Glutamax (Thermo Fisher). Cells (monolayer) were then seeded on poly-D-lysine $(100 \mu \mathrm{g} / \mathrm{mL}$, Sigma Aldrich) and laminin (10 $\mu \mathrm{g} / \mathrm{mL}$, Sigma Aldrich) coated coverslips at a density of $2 \times 10^{4}$ cells $/ \mathrm{cm}^{2}$ (Teixeira et al. 2015). Intact neurospheres were directly added on coated coverslips without dissociation. "Intact neurosphere seeding was only performed for an initial visual evaluation. All other experiments are performed with monolayer cells obtained from dissociated neurospheres." Memantine treatment was performed by dissolving memantine (Sigma Aldrich) in PBS and adding to media at a final concentration of $2 \mu \mathrm{M}$ (Jantas and Lason 2009). Final vehicle concentration in media was kept below $0.01 \%$. Sham and ELF MF group plates were placed in different incubators at $37{ }^{\circ} \mathrm{C}$ under $5 \% \mathrm{CO}_{2}$ atmosphere. Cells were allowed to differentiate for 5 days under control (sham exposure) or continuous 50-Hz, 1.0-mT ELF MF exposure conditions. ELF MF exposure was provided by a Helmholtz coil pair placed inside the cell culture incubator (Cho et al. 2012). 50-Hz sinusoidal signal was obtained by attenuating power line signal through a variac and fed to the coils to maintain a $1.0 \mathrm{mT}$ root mean square field intensity which was measured by an EMF spectrum analyzer (Aaronia NF-5035).

\section{Immunocytochemistry}

hNPCs were washed with PBS and fixed with $4 \%$ formaldehyde (Sigma Aldrich) solution in PBS for $10 \mathrm{~min}$ and permeabilized with PBS containing 0.1\% Triton X-100 (Sigma Aldrich). After blocking non-specific binding sites with $10 \%$ fetal calf serum (Thermo Fisher) in PBS for $1.0 \mathrm{~h}$ at room temperature, coverslips were incubated in primary antibodies against MAP2 (mature neuronal marker Abcam, mouse, 1:500), doublecortin (DCX) (Santa Cruz, goat, 1:300) or c-fos (Abcam, Rabbit, 1:300) for $2.0 \mathrm{~h}$ at room temperature. Corresponding secondary antibodies (Abcam, 1:500) conjugated with either Alexa Fluor 488 or Alexa Fluor 594 were sequentially added and incubated at room temperature for $1.0 \mathrm{~h}$. Nuclei were counterstained with 4-6-diamidino2-phenylindole-dihydrochloride (DAPI) for $10 \mathrm{~min}$ and micrographs were taken with fluorescence microscopy (Olympus BX-61) (Teixeira et al. 2016). 


\section{Western blot}

Differentiated hNPCs were harvested into ice-cold RIPA buffer and protein concentrations were estimated by Bradford assay (Bio-Rad). Lysates were diluted in 2X Laemmli buffer before use. $4-10 \%$ discontinuous polyacrylamide (Sigma Aldrich) gels were cast and proteins were resolved under $120 \mathrm{~V}$ (Bio-Rad, Mini Protean). Protein bands were transferred (Bio-Rad, Transblot Turbo) to PVDF membranes (Millipore). Membranes were probed with primary antibodies against MAP2 (Abcam, mouse, 1:500), Tuj1 (Santa Cruz, mouse, 1:1000) and DCX (Abcam, rabbit, 1:500) at $4{ }^{\circ} \mathrm{C}$, overnight. Corresponding horseradish peroxidase conjugated secondary antibodies (Abcam, 1:2000) were added on membranes and incubated for $1.0 \mathrm{~h}$ at room temperature. Bands were visualized by adding 3,3',5,5'-tetramethylbenzidine chromogenic substrate (Thermo Fisher) on the membranes. Scanned membranes were quantified by ImageJ software.

\section{Quantification of micrographs}

ImageJ software was used for analyzing immunostaining micrographs. Marker positive cells were counted by applying a threshold to each of at least 25 images from each group, so that only marker-expressing cells remain on the foreground. DAPI stained total nuclei and cells positive for each marker were counted manually.

NeuronJ, an ImageJ plugin, (Jacob et al. 2004) was used to manually mark and measure the length of all MAP2 ${ }^{+}$ neurites from at least 20 images from each group.

Micrographs from c-fos immunostaining experiment were converted to grayscale and each $\mathrm{MAP}^{+}$cell was manually selected with region of interest tool for making mean gray value measurements for c-fos. Background measurements were subtracted from intensity values of each cell. At least 19 images were analyzed for each group.

\section{Statistical analysis}

Statistical significance of ELF MF exposure and memantine treatment effects were tested by two-way ANOVA followed by Tukey's HSD post hoc test using Graphpad Prism software. One-way ANOVA was used for cell counts where memantine treatment was not performed. All data presented as mean \pm SEM of three independent experiments. Calculated $p$ values that are lower than 0.05 were considered significant and denoted as * for $p<0.05$, ** for $p<0.01$ and n.d. for differences that are not significant. Exact $\mathrm{p}$ values are given in the text.

\section{Results}

The effect of ELF MF exposure on neuronal differentiation of hNPCs was first assessed by immunostaining for early (DCX, marker for immature neurons) and mature neurons (MAP2). Intact neurospheres and dissociated cells were differentiated for qualitative and quantitative evaluation respectively, under sham and continuous ELF MF $(50 \mathrm{~Hz}$, $1.0 \mathrm{mT}$ ) exposure conditions. hNPCs differentiated as a monolayer resulted in a population of $52.5 \% \pm 2.9 \mathrm{DCX}^{+}$ and $57.0 \% \pm 1.82 \mathrm{MAP}^{+}$cells. Total number of cells and populations did not differ ( $p=0.899$ and 0.507 for DCX and MAP2, respectively) under ELF MF exposure conditions (Fig. 1a). Neurospheres attached to the culture surface upon induction of differentiation and cells started to migrate. A gradual decline of $\mathrm{DCX}^{+}$was observed as cells migrated away from the neurosphere core; whereas, ELF MF group showed an abrupt reduction in $\mathrm{DCX}^{+}$cells immediately outside the neurosphere boundary (Fig. 1c). This finding was expressed as the profile of DCX signal intensity plotted against the distance from neurosphere core (Fig. 1b). DCX signal at the neurosphere center decays to $10 \%$ of initial values within $259 \pm 47 \mu \mathrm{m}$ distance in ELF MF group, compared to $482 \pm 30 \mu \mathrm{m}$ in sham group $(p=0.04)$.

Neuronal differentiation of monolayer cultures was further evaluated through total abundances of neuronal markers, comparatively estimated by western blots. Overall results of two-way ANOVA reveal significant effect of ELF MF on all tested markers ( $p=0.003,0.013$ and 0.042 for TUJ1, MAP2 and DCX, respectively) but no significant effect of memantine treatment alone $(p=0.434,0.193$ and 0.762 for TUJ1, MAP2 and DCX, respectively). ELF MF exposure resulted in significantly $(p=0.016$ and $p=0.003$ respectively) elevated total levels of mature neuronal markers MAP2 and TUJ1 (Fig. 2a and b), while early neuron and migration marker DCX levels showed a sharp drop $(p=0.009)$ compared to sham exposure (Fig. 2c). Blocking NMDA receptors, by treating the cells with uncompetitive channel antagonist memantine, neutralized the effects of ELF MF exposure on all three markers. Memantine treatment alone had little to no effect on $(p=0.323,0.859$ and 0.076 for TUJ1, MAP2 and DCX, respectively) the abundance of markers (Fig. 2a, b, c).

ELF MF also enhanced morphological maturation of neurons in an NMDA receptor dependent manner. Overall, two-way ANOVA results show significant effects for both $\operatorname{ELF} \operatorname{MF}(p=0.007)$ and memantine treatment $(p<0.001)$ on neurite lengths of mature neurites. MAP2 ${ }^{+}$neurites were significantly longer $(p=0.004)$ in cells differentiated under exposure while memantine treated cells were not $(p=0.949)$ affected by ELF MF (Fig. 3a) according to post hoc analysis. An NMDA receptor-based effect of ELF MF on neuronal 
a

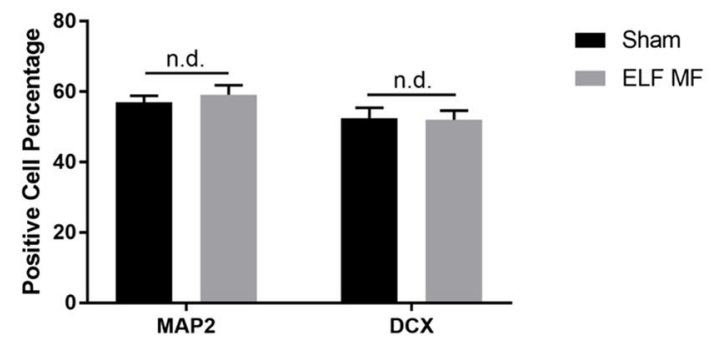

b

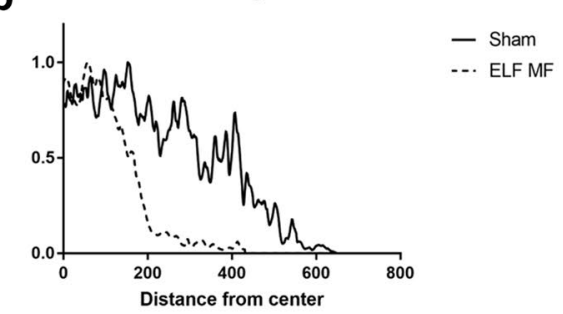

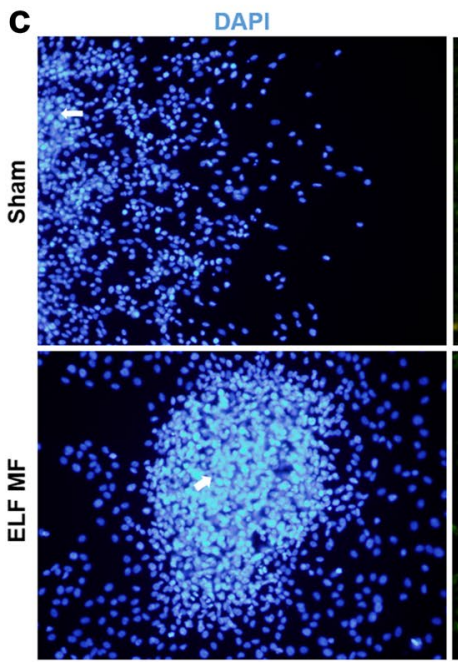

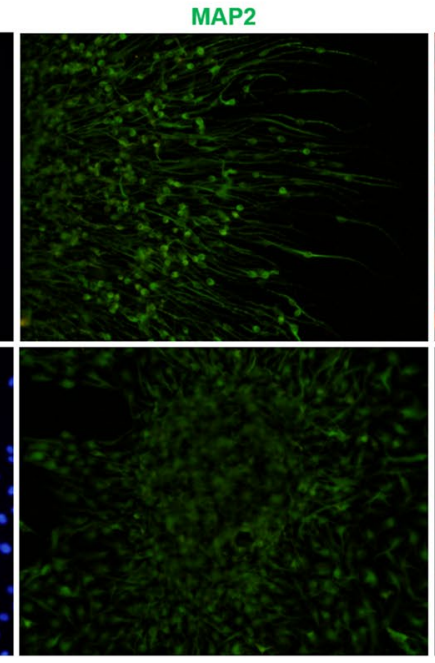

DCX

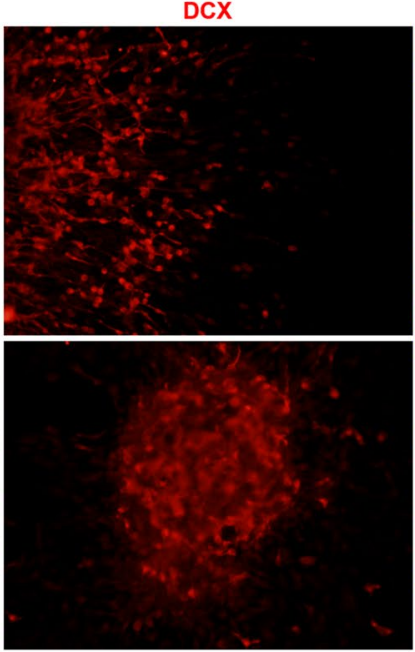

DAPI MAP2 DCX

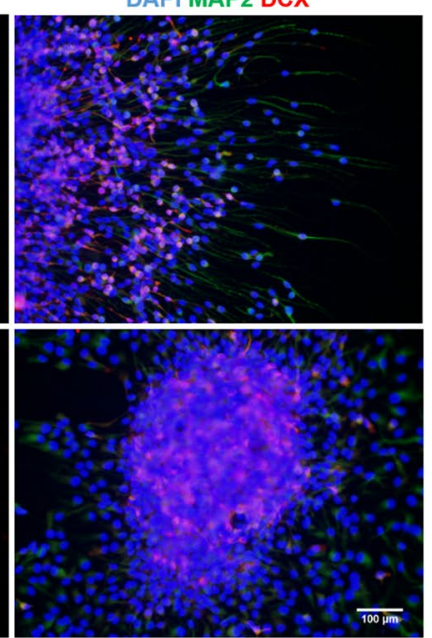

Fig. 1 Assessment of immature $\left(\mathrm{DCX}^{+}\right)$and mature $\left(\mathrm{MAP}^{+}\right)$neuron populations under 50-Hz, 1.0-mT ELF MF exposure a Quantification of $\mathrm{MAP}^{+}$and $\mathrm{DCX}^{+}$neurons obtained from monolayer cultures as a percentage of total nuclei under sham and ELF MF conditions $\mathbf{b}$ Sample DCX intensity profiles of images taken from neurons differentiated as intact neurospheres as a function of distance from the neu-

activity was also identified by measuring c-fos levels in $\mathrm{MAP}^{+}$cells. Mean c-fos fluorescence signal intensities within these cells were heightened by both ELF MF exposure $(0.007)$ and memantine treatment $(p<0.001)$ according to the overall two-way ANOVA test. Presence of memantine alone produced a sharp increase $(p<0.001)$ in c-fos levels but also eliminated the effect of ELF MF exposure as signal intensities were indistinguishable between sham and ELF MF groups under memantine treatment (Fig. 3b).

\section{Discussion}

Despite concerns about biological effects of chronic exposure and its epidemiological association with neurodegenerative diseases, (Consales et al. 2012) ELF MFs may hold potential for new, non-invasive intervention options for diseases of neural tissues due to their unperturbed penetration into biological media and subtle but promising effects on the molecular level. These include ameliorating cognitive symptoms of Alzheimer's disease (Arendash et al. 2010; Liu et al. 2015) and attenuating tau phosphorylation which rosphere center c DCX (red) and MAP2 (green) immunofluorescence images of neurons differentiated as intact neurospheres where initial neurosphere cores are marked with white arrows on DAPI (blue) stained nuclei; scale bar $100 \mu \mathrm{m}$ for all micrographs in panel. Data presented as the mean \pm SEM of three independent experiments; nd non-significant according to one-way ANOVA

is one of the molecular hallmarks of Alzheimer disease (Hu et al. 2016). They are known to drive different stem cell populations towards neural differentiation when applied at $50 \mathrm{~Hz}$, in vitro (Cho et al. 2012; Park et al. 2013; Ma et al. 2014, 2016) and in vivo (Cuccurazzu et al. 2010), even in the absence of differentiation factors (Cho et al. 2012). Although investigations into neural differentiation effects almost exclusively use $50 \mathrm{~Hz}$ fields, other parameters such as field intensity, exposure duration, exposure intervals and signal waveform vary widely between studies as well as their obtained results (Cui et al. 2017). Optimizing these parameters with a purposefully designed waveform to elicit a selective, well-defined and useful molecular response constitutes the ultimate goal of this field. Pinpointing exact interaction mechanisms of ELF MF with neural tissue is therefore the major milestone towards this target.

The absence of an effect of ELF MF on $\mathrm{MAP}^{+}$cell population is already expected since neuronal-glial fate is determined by completely different processes which cannot be altered during differentiation after commitment to a cell type (Sanalkumar et al. 2010). However, intact neurospheres seem to dissipate less efficiently under 
a

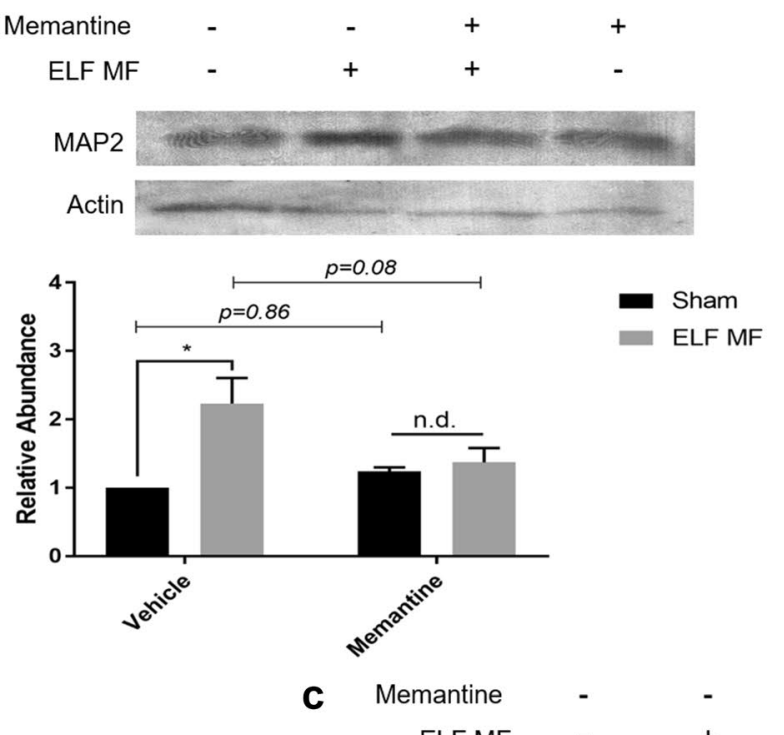

b Memantine
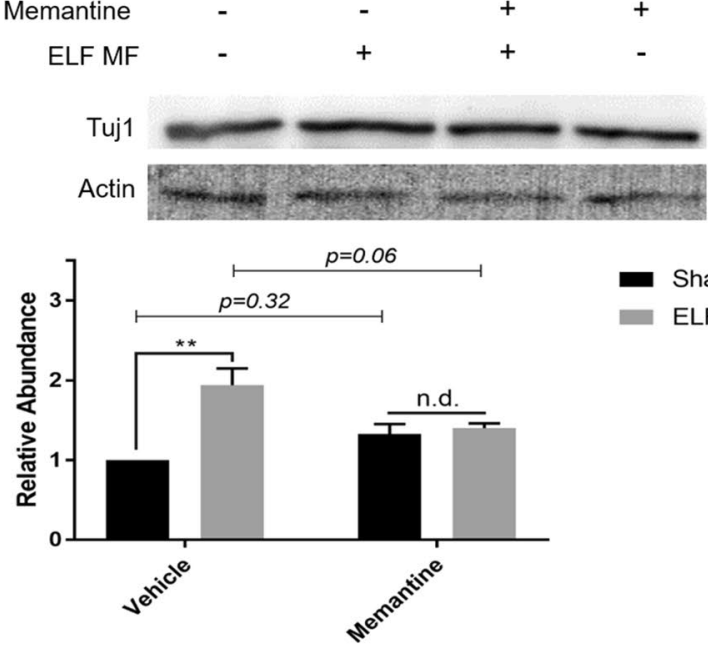

- Sham - ELF MF

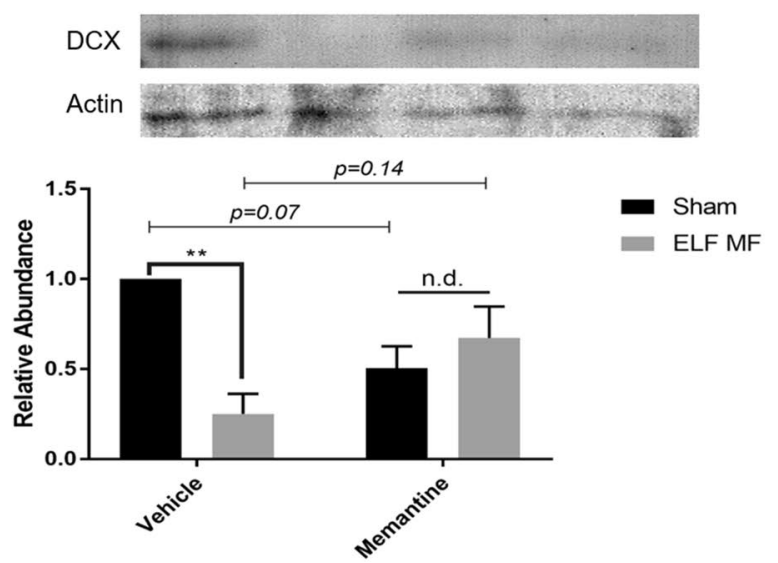

Fig. 2 Western blot analysis of early and mature neuron markers in monolayer cultures under ELF MF exposure and memantine treatment conditions. Representative blot images and densitometry analyses of a MAP2, b TUJ1 and $\mathbf{c}$ DCX as normalized to actin expression. Data presented as the mean \pm SEM of three independent experiments; $* * p<0.01, * p<0.05$, nd non-significant according to Tukey's HSD post hoc test

channel block, meaning glutamate binding and $\mathrm{Mg}^{2+}$ block dislodging are both required for memantine activity, which also make it a voltage-dependent blocker. After memantine binds to channel opening, the channel can close and trap the molecule inside. This characteristic is shared with other common NMDA receptor blockers such as amantadine, ketamine, MK-801 and phencyclidine (Johnson and Kotermanski 2006; Parsons and Gilling 2007; Song et al. 2018). Our results demonstrate that blocking NMDA receptors in this manner prevents ELF MF from enhancing neuronal marker levels as well as restoring early neuron marker DCX to normal levels. These findings create adequate basis to propose that exposure to ELF MF magnifies NMDA receptor activation which leads to improvement of neuronal differentiation. It can be speculated that ELF MF may increase channel open probability through 

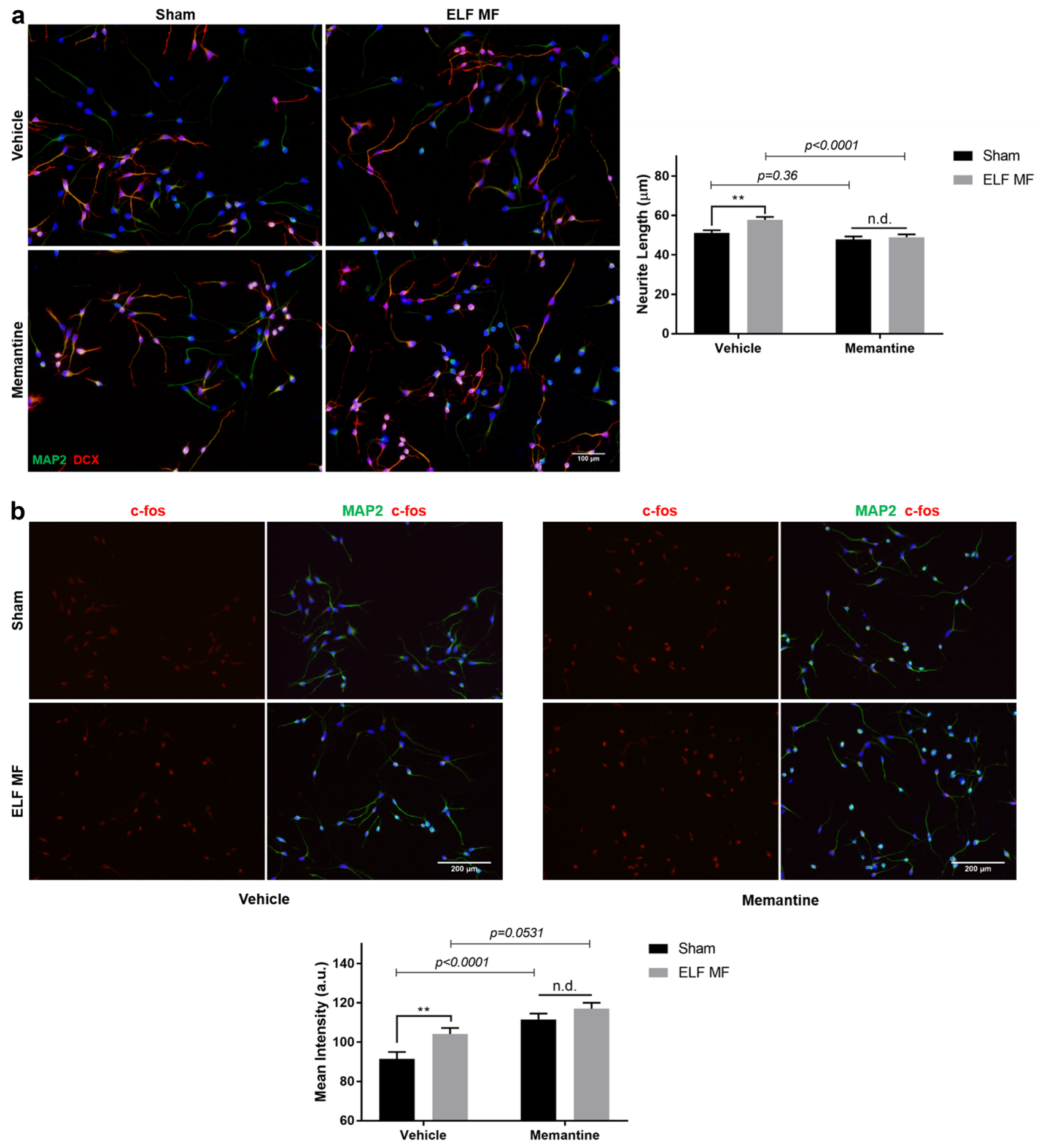

Fig. 3 Effect of ELF MF exposure and memantine treatment on neurite lengths and c-fos expression of hNPCs differentiated as monolayers. a Representative images of MAP2 (green) and DCX (red) immunostained cells along with neurite length analysis of each group $\mathbf{b}$ Representative images of hNPCs immunostained for MAP2 (green)

effects on the $\mathrm{Mg}^{2+}$ block or due to local fluctuations in the trans-membrane potential caused by induced eddy currents. We further reinforced our findings by analyzing and c-fos (red) given with quantification analysis of c-fos signal intensity from MAP2 $2^{+}$cells. Data presented as the mean \pm SEM of three independent experiments; $* * p<0.01, * p<0.05$, n.d. non-significant according to Tukey's HSD post hoc test

two more NMDA receptor-facilitated events; c-fos levels (Lerea et al. 2018) and neurite outgrowth (Pearce et al. 1987; Hong et al. 2015; Cambray-Deakin and R.D. 1990). 
An increase in c-fos expression of mature neurons was already expected as a response to the rise in intracellular $\mathrm{Ca}^{2+}$ levels which confirms previous studies reporting $\mathrm{Ca}^{2+}$ influx in response to ELF MF exposure (Walleczek 1992; Karabakhtsian et al. 1994; Morgado-Valle et al. 1998; Fanelli et al. 1999; Zhou et al. 2002; Lisi et al. 2006; Manikonda et al. 2007; Sun et al. 2016). Interestingly, presence of memantine alone also resulted in significant increase in c-fos which is explained by previous findings where memantine administration induced c-fos expression in cortical neurons in vivo (De Bartolomeis et al. 2013). Conversely, memantine treated cells exhibited similar levels of c-fos between sham and ELF MF exposure groups, eliciting that $\mathrm{Ca}^{2+}$ influx under exposure is triggered by NMDA receptors. Lastly, we show that neurite outgrowth is augmented by ELF MF exposure, confirming previously reported results (Ma et al. 2016), which was also shown to be an NMDA receptor-mediated effect as displayed by relapsed neurite lengths in memantine treated group.

Overall, our results show that ELF MF exposure amplifies neuronal differentiation of hNPCs in an NMDA receptor-dependent manner. Other previously reported effects such as elevation of c-fos levels, intracellular $\mathrm{Ca}^{2+}$ and elongated neurites are also demonstrated to be facilitated by NMDA receptor activation. These findings allow us to postulate that effects of ELF MF on neural differentiation are initiated by its effects on activation mechanisms of NMDA receptors. In essence, this work identifies NMDA receptors as a strong candidate for future studies which pursues interaction mechanisms of ELF MF with neural tissues.

Funding A. Ozgun was supported within the framework of European Molecular Biology Organization Short Term Fellowships, ASTF 7502. This work was partially supported by TUBITAK Projects under Grant No. 117Z864, Bogazici University Research Fund by Grant Number 6701. A.J. Salgado and A. Marote acknowledge the financial support from: Prémios Santa Casa Neurociências-Prize Melo e Castro for Spinal Cord Injury Research (MC-04/17); Portuguese Foundation for Science and Technology Pre-Doctoral fellowship to A. Marote PDE/ BDE/113598/2015 and IF Development Grant to A. J. Salgado. This work is funded by national funds through FCT under the scope of grant reference TUBITAK/0007/2014. This article has been developed under the scope of the projects NORTE-01-0145-FEDER-000023, supported by the Northern Portugal Regional Operational Programme (NORTE 2020), under the Portugal 2020 Partnership Agreement, through the European Regional Development Fund (FEDER). This work has been funded by FEDER funds, through the Competitiveness Factors Operational Programme (COMPETE), and by National funds, through the Foundation for Science and Technology (FCT), under the scope of the project POCI-01-0145-FEDER-007038 and POCI-01-0145-FEDER-029206.

\section{Compliance with ethical standards}

Conflict of interest Authors declare no conflict of interest.

\section{References}

Anikeeva P (2016) Optogenetics unleashed. Nat Biotechnol 34:4344. https://doi.org/10.1038/nbt.3458

Anikeeva P, Jasanoff A (2016) Problems on the back of an envelope. Elife. https://doi.org/10.7554/elife.19569

Arendash GW, Sanchez-Ramos J, Mori T et al (2010) Electromagnetic field treatment protects against and reverses cognitive impairment in Alzheimer's disease mice. J Alzheimers Dis 19:191-210. https://doi.org/10.3233/JAD-2010-1228

Ayanlaja AA, Xiong Y, Gao Y et al (2017) Distinct features of doublecortin as a marker of neuronal migration and its implications in cancer cell mobility. Front Mol Neurosci. https://doi. org/10.3389/fnmol.2017.00199

Baek S, Quan X, Kim S et al (2014) Electromagnetic fields mediate efficient cell reprogramming into a pluripotent state. ACS Nano 8:10125-10138. https://doi.org/10.1021/nn502923s

Baghbaderani BA, Mukhida K, Sen A et al (2010) Bioreactor expansion of human neural precursor cells in serum-free media retains neurogenic potential. Biotechnol Bioeng 5:5. https://doi. org/10.1002/bit.22590

Boorman GA, Gauger JR, Johnson TR et al (1997) Eight-week toxicity study of $60 \mathrm{~Hz}$ magnetic fields in $\mathrm{F} 344$ rats and B6C3F1 mice. Toxicol Sci 35:55-63. https://doi.org/10.1093/toxsc i/35.1.55

Cambray-Deakin MA, Burgoyne RD (1990) Regulation of neurite outgrowth from cerebellar granule cells in culture: nmda receptors and protein kinase C. Excitatory amino acids and neuronal plasticity. Advances in experimental medicine and biology. Springer, Boston

Chang H-R, Kuo C-C (2008) The activation gate and gating Mechanism of the NMDA receptor. J Neurosci. https://doi. org/10.1523/jneurosci.3485-07.2008

Cho H, Seo Y-K, Yoon H-H et al (2012) Neural stimulation on human bone marrow-derived mesenchymal stem cells by extremely low frequency electromagnetic fields. Biotechnol Prog 28:1329-1335. https://doi.org/10.1002/btpr.1607

Choi Y-K, Lee DH, Seo Y-K et al (2014) Stimulation of neural differentiation in human bone marrow mesenchymal stem cells by extremely low-frequency electromagnetic fields incorporated with MNPs. Appl Biochem Biotechnol 174:1233-1245. https ://doi.org/10.1007/s12010-014-1091-z

Chow BY, Boyden ES (2013) Optogenetics and translational medicine. Sci Transl Med 5:177ps5. https://doi.org/10.1126/scitr anslmed.3003101

Collingridge GL, Watkins JC (1994) The NMDA receptor. Oxford University Press. ISBN: 9780192625021

Consales C, Merla C, Marino C, Benassi B (2012) Electromagnetic fields, oxidative stress, and neurodegeneration. Int J Cell Biol. https://doi.org/10.1155/2012/683897

Consales C, Cirotti C, Filomeni G et al (2018) Fifty-hertz magnetic field affects the epigenetic modulation of the mir-34b/c in neuronal cells. Mol Neurobiol. https://doi.org/10.1007/s12035-017-0791-0

Cuccurazzu B, Leone L, Podda MV et al (2010) Exposure to extremely low-frequency $(50 \mathrm{~Hz})$ electromagnetic fields enhances adult hippocampal neurogenesis in C57BL/6 mice. Exp Neurol 226:173182. https://doi.org/10.1016/j.expneurol.2010.08.022

Cui M, Ge H, Zhao H et al (2017) Electromagnetic fields for the regulation of neural stem cells. Stem Cells Int. https://doi. org/10.1155/2017/9898439

Davanipour Z, Tseng CC, Lee PJ, Sobel E (2007) A case-control study of occupational magnetic field exposure and Alzheimer's disease: results from the california alzheimer's disease diagnosis and treatment centers. BMC Neuro. https://doi. org/10.1186/1471-2377-7-13 
De Bartolomeis A, Sarappa C, Buonaguro EF et al (2013) Different effects of the NMDA receptor antagonists ketamine, MK-801, and memantine on postsynaptic density transcripts and their topography: role of homer signaling, and implications for novel antipsychotic and pro-cognitive targets in psychosis. Prog NeuroPsychopharmacol Biol Psychiatry. https://doi.org/10.1016/j.pnpbp .2013.06.010

Fanelli C, Coppola S, Barone R et al (1999) Magnetic fields increase cell survival by inhibiting apoptosis via modulation of $\mathrm{Ca}^{+}$ influx. FASEB J 13:95-102

Gilbert F, Harris AR, Kapsa RMI (2014) Controlling brain cells with light: ethical considerations for optogenetic clinical Trials. AJOB Neurosci 5:3-11. https://doi.org/10.1080/21507740.2014.911213

Hardwick RM, Lesage E, Miall RC (2014) Cerebellar transcranial magnetic stimulation: the role of coil geometry and tissue depth. Brain Stimul 7:643-649. https://doi.org/10.1016/j.brs.2014.04.009

Hong F, Sheng L, Ze Y et al (2015) Suppression of neurite outgrowth of primary cultured hippocampal neurons is involved in impairment of glutamate metabolism and NMDA receptor function caused by nanoparticulate $\mathrm{TiO}_{2}$. Biomaterials. https://doi. org/10.1016/j.biomaterials.2015.02.067

Hu Y, Lai J, Wan B et al (2016) Long-term exposure to ELF-MF ameliorates cognitive deficits and attenuates tau hyperphosphorylation in 3xTg AD mice. Neurotoxicology. https://doi.org/10.1016/j. neuro.2016.02.012

Iacobucci GJ, Popescu GK (2017) NMDA receptors: linking physiological output to biophysical operation. Nat Rev Neurosci 18:236-249

Iseri E, Kuzum D (2017) Implantable optoelectronic probes for in vivo optogenetics. J Neural Eng 14:31001. https://doi. org/10.1088/1741-2552/aa60b3

Jacob M, Unser M, Sarria J-CF et al (2004) Design and validation of a tool for neurite tracing and analysis in fluorescence microscopy images. Cytometry 58A:167-176. https://doi.org/10.1002/ cyto.a.20022

Jansson LC, Akerman KE (2014) The role of glutamate and its receptors in the proliferation, migration, differentiation and survival of neural progenitor cells. J Neural Transm. https://doi.org/10.1007/ s00702-014-1174-6

Jantas D, Lason W (2009) Protective effect of memantine against doxorubicin toxicity in primary neuronal cell cultures: influence a development stage. Neurotox Res. https://doi.org/10.1007/s1264 0-009-9002-8

Johnson JW, Kotermanski SE (2006) Mechanism of action of memantine. Curr Opin Pharmacol 6(1):61-67. https://doi.org/10.1016/j. coph.2005.09.007

Joo J-Y, Kim B-W, Lee J-S et al (2007) Activation of NMDA receptors increases proliferation and differentiation of hippocampal neural progenitor cells. J Cell Sci 120:1358-1370. https://doi. org/10.1242/jcs.002154

Karabakhtsian R, Broude N, Shalts N et al (1994) Calcium is necessary in the cell response to EM fields. FEBS Lett 349:1-6. https://doi. org/10.1016/0014-5793(94)00618-0

Kazemi M, Sahraei H, Aliyari H et al (2018) Effects of the extremely low frequency electromagnetic fields on NMDA-receptor gene expression and visual working memory in male rhesus macaques. Basic Clin Neurosci J 9:167-176. https://doi.org/10.29252/nirp. bcn.9.3.167

Kim H-J, Jung J, Park J-H et al (2013) Extremely low-frequency electromagnetic fields induce neural differentiation in bone marrow derived mesenchymal stem cells. Exp Biol Med (Maywood) 238:923-931. https://doi.org/10.1177/1535370213497173

Klomjai W, Katz R, Lackmy-Vallée A (2015) Basic principles of transcranial magnetic stimulation (TMS) and repetitive TMS (rTMS). Ann Phys Rehabil Med 58:208-213
Lerea L, Butler L, McNamara J (2018) NMDA and non-NMDA receptor-mediated increase of c-fos mRNA in dentate gyrus neurons involves calcium influx via different routes. J Neurosci. https:// doi.org/10.1523/jneurosci.12-08-02973.1992

Li C, Xie M, Luo F et al (2014) The extremely low-frequency magnetic field exposure differently affects the AMPAR and NMDAR subunit expressions in the hippocampus, entorhinal cortex and prefrontal cortex without effects on the rat spatial learning and memory. Environ Res 134:74-80. https://doi.org/10.1016/j.envre s.2014.06.025

Lisi A, Ledda M, Rosola E et al (2006) Extremely low frequency electromagnetic field exposure promotes differentiation of pituitary corticotrope-derived AtT20 D16 V cells. Bioelectromagnetics 27:641-651. https://doi.org/10.1002/bem.20255

Liu X, Zuo H, Wang D et al (2015) Improvement of spatial memory disorder and hippocampal damage by exposure to electromagnetic fields in an Alzheimer's disease rat model. PLoS One. https://doi. org/10.1371/journal.pone.0126963

Long X, Zhang S-J (2018) Commentary: MagR alone is insufficient to confer cellular calcium responses to magnetic stimulation. Front Neural Circuits. https://doi.org/10.3389/fncir.2018.00097

Ma Q, Deng P, Zhu G et al (2014) Extremely low-frequency electromagnetic fields affect transcript levels of neuronal differentiationrelated genes in embryonic neural stem cells. PLoS One. https:// doi.org/10.1371/journal.pone.0090041

Ma Q, Chen C, Deng P et al (2016) Extremely low-frequency electromagnetic fields promote in vitro neuronal differentiation and neurite outgrowth of embryonic neural stem cells via up-regulating TRPC1. PLoS One 11:e0150923. https://doi.org/10.1371/journ al.pone. 0150923

Manikonda PK, Rajendra P, Devendranath D et al (2007) Influence of extremely low frequency magnetic fields on $\mathrm{Ca}^{2+}$ signaling and NMDA receptor functions in rat hippocampus. Neurosci Lett 413:145-149. https://doi.org/10.1016/j.neulet.2006.11.048

Maziarz A, Kocan B, Bester M et al (2016) How electromagnetic fields can influence adult stem cells: positive and negative impacts. Stem Cell Res Ther 7:54. https://doi.org/10.1186/s13287-016-0312-5

Mendez I, Dagher A, Hong M et al (2002) Simultaneous intrastriatal and intranigral fetal dopaminergic grafts in patients with Parkinson disease: a pilot study. Report of three cases. J Neurosurg 96:589-596. https://doi.org/10.3171/jns.2002.96.3.0589

Morgado-Valle C, Verdugo-Diaz L, Garcia DE et al (1998) The role of voltage-gated $\mathrm{Ca}^{2+}$ channels in neurite growth of cultured chromaffin cells induced by extremely low frequency (ELF) magnetic field stimulation. Cell Tissue Res 291:217-230

Nikolova T, Czyz J, Rolletschek A et al (2005) Electromagnetic fields affect transcript levels of apoptosis-related genes in embryonic stem cell-derived neural progenitor cells. FASEB J 19:1686-1688. https://doi.org/10.1096/fj.04-3549fje

Nimpf S, Keays DA, Chen R et al (2017) Is magnetogenetics the new optogenetics? EMBO J 36:602-606. https://doi.org/10.15252/ EMBJ.201797177

Papadia S, Hardingham GE (2007) The dichotomy of NMDA receptor signaling. Neuroscientist 13:572-579. https://doi. org/10.1177/10738584070130060401

Park J-E, Seo Y-K, Yoon H-H et al (2013) Electromagnetic fields induce neural differentiation of human bone marrow derived mesenchymal stem cells via ROS mediated EGFR activation. Neurochem Int 62:418-424. https://doi.org/10.1016/j.neuin t.2013.02.002

Parsons CG, Gilling K (2007) Memantine as an example of a fast, voltage-dependent, open channel N-methyl-D-aspartate receptor blocker. Methods Mol Biol 403:15-36

Pearce IA, Cambray-Deakin MA, Burgoyne RD (1987) Glutamate acting on NMDA receptors stimulates neurite outgrowth from 
cerebellar granule cells. FEBS Lett. https://doi.org/10.1016/00145793(87)80525-2

Pettenati MJ, George SK, Cathey L et al (2018) Evaluation of cytotoxic and genotoxic effects of extremely low-frequency electromagnetic field on mesenchymal stromal cells. Glob Adv Heal Med 7:216495611877747. https://doi.org/10.1177/2164956118777472

Qin S, Yin H, Yang C et al (2015) A magnetic protein biocompass. Nat Mater 15:217-226. https://doi.org/10.1038/nmat4484

Saliev T, Mustapova Z, Kulsharova G et al (2014) Therapeutic potential of electromagnetic fields for tissue engineering and wound healing. Cell Prolif 47:485-493

Salvador R, Miranda PC, Roth Y, Zangen A (2007) High-permeability core coils for transcranial magnetic stimulation of deep brain regions. In: Annual International Conference of the IEEE Engineering in Medicine and Biology_Proceedings. pp 6652-6655

Sanalkumar R, Vidyanand S, Lalitha Indulekha C, James J (2010) Neuronal vs. Glial fate of embryonic stem cell-derived neural progenitors (ES-NPs) is determined by FGF2/EGF during proliferation. J Mol Neurosci. https://doi.org/10.1007/s12031-010-9335-Z

Seong Y, Moon J, Kim J (2014) Egr1 mediated the neuronal differentiation induced by extremely low-frequency electromagnetic fields. Life Sci 102:16-27. https://doi.org/10.1016/j.lfs.2014.02.022

Song X, Jensen MO, Jogini V et al (2018) Mechanism of NMDA receptor channel block by MK-801 and memantine. Nature 556:515519. https://doi.org/10.1038/s41586-018-0039-9

Stanley SA, Sauer J, Kane RS et al (2014) Remote regulation of glucose homeostasis in mice using genetically encoded nanoparticles. Nat Med 21:92-98. https://doi.org/10.1038/nm.3730

Sun Z-C, Ge J-L, Guo B et al (2016) Extremely low frequency electromagnetic fields facilitate vesicle endocytosis by increasing presynaptic calcium channel expression at a central synapse. Nat Publ 5:1-11. https://doi.org/10.1038/srep21774

Tang S-J, Yang X-L, Li B et al (2012) Activation of NMDA receptors upregulates a disintegrin and metalloproteinase 10 via a Wnt/ MAPK signaling pathway. J Neurosci 5:5. https://doi.org/10.1523/ jneurosci.3916-11.2012

Teixeira FG, Panchalingam KM, Anjo SI et al (2015) Do hypoxia/normoxia culturing conditions change the neuroregulatory profile of Wharton Jelly mesenchymal stem cell secretome? Stem Cell Res Ther. https://doi.org/10.1186/s13287-015-0124-Z

Teixeira FG, Panchalingam KM, Assunção-Silva R et al (2016) Modulation of the mesenchymal stem cell secretome using computer-controlled bioreactors: impact on neuronal cell proliferation, survival and differentiation. Sci Rep. https://doi. org/10.1038/srep27791

Temme L, Schepmann D, Schreiber JA et al (2018) Comparative pharmacological study of common NMDA receptor open channel blockers regarding their affinity and functional activity toward GluN2A and GluN2B NMDA receptors. Chem Med Chem 13:446-452. https://doi.org/10.1002/cmdc.201700810

Blanke ML, VanDongen AMJ. Activation mechanisms of the NMDA receptor. In: Van Dongen AM, editor. Biology of the NMDA receptor, chapter 13. Boca Raton: CRC Press/Taylor \& Francis; 2009

Walleczek J (1992) Electromagnetic field effects on cells of the immune system: the role of calcium signalling. Faseb 6:3177-3185

Wheeler MA, Smith CJ, Ottolini M et al (2016) Genetically targeted magnetic control of the nervous system. Nat Neurosci Adv. https ://doi.org/10.1038/nn.4265

Yoneyama M, Nakamichi N, Fukui M et al (2008) Promotion of neuronal differentiation through activation of $N$-methyl-D-aspartate receptors transiently expressed by undifferentiated neural progenitor cells in fetal rat neocortex. J Neurosci Res. https://doi. org/10.1002/jnr.21696

Yu JZ, Wu H, Yang Y et al (2014) Osteogenic differentiation of bone mesenchymal stem cells regulated by osteoblasts under EMF exposure in a co-culture system. J Huazhong Univ Sci Technol Med Sci 34:247-253. https://doi.org/10.1007/s11596-014-1266-4

Zhou J, Yao G, Zhang J, Chang Z (2002) CREB DNA binding activation by a $50-\mathrm{Hz}$ magnetic field in HL60 cells is dependent on extra- and intracellular $\mathrm{Ca}^{2+}$ but not PKA, PKC, ERK, or p38 MAPK. Biochem Biophys Res Commun 296:1013-1018. https ://doi.org/10.1016/S0006-291X(02)02022-3

Zhou H, Chen G, Chen C et al (2012) Association between extremely low-frequency electromagnetic fields occupations and amyotrophic lateral sclerosis: a meta-analysis. PLoS One. https://doi. org/10.1371/journal.pone.0048354

Publisher's Note Springer Nature remains neutral with regard to jurisdictional claims in published maps and institutional affiliations. 